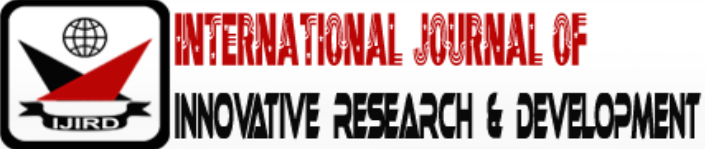

ISSN 2278 - 0211 (Online)

\section{Geoenvironmental Investigation of Some Physical and Chemical Constituents of Ground Waters in of Sapele and Environs, Western Niger Delta, Nigeria}

Izeze Elijah Ovie
Lecturer, Department of Earth Sciences,
Federal University of Petroleum Resources, Effurun, Nigeria
Imasuen Isaac Okpeseyi
Professor, Department of Geology,
University of Benin, Benin City, Nigeria
Ighodaro Ehika Joseph
Lecturer, Department of Geology and Petroleum Studies,
Western Delta University, Oghara, Delta State, Nigeria

\begin{abstract}
:
Hydrogeochemical studies have been carried out in parts of Sapele and Warri Local Government Areas of Delta State in order to determine the different hydrogeochemical facies and understand the groundwater flow regime and its effect on the chemical composition of the groundwater so as to evaluate the anthropogenic effects on groundwater quality in the study area. Twenty-five (25) water samples were taken from Sapele (18) and Warri (7) in one liter plastic cans with temperature, $\mathrm{pH}$, electrical conductivity and Total Dissolved Solids (TDS) determined in-situ. The samples were then taken to the laboratory where the analyses were done using the Varian 220 flame atomic absorption spectrophotometer. Salinity and chloride in water was carried out in accordance with the American Petroleum Institute (API-RP) 45. Phosphate, Nitrate, Sulphate, Calcium, Potassium, Sodium, Magnesium and Ammonium were all determined in accordance with the American Public Health Association (APHA) 425C,427C and 417C.

The analyses carried out indicated the presence of chloride ion as the dominant parameter. Chloride shows a negative correlation with all other parameters thus indicating that its source is from surface saline waters close to those areas with high chloride concentrations. The presence of ammonia in such high concentrations and its strong positive correlation with phosphate supports that its source is as a result of pollution from sewage. The positive correlation of phosphate, sulphate, nitrate and TDS also indicate pollution from sewage. GPS readings and computation of several hydrogeological parameters showed a SW direction of groundwater flow for Sapele and a SE flow direction for Warri. The presence of ammonium at levels higher than geogenic (geologically related processes) levels is a strong indicator of fecal pollution. The groundwater in the area is mostly acidic with high chloride concentrations.

Contamination from anthropogenic sources is mainly as a result of waste waters from brines released as by-products of oil exploration activities and leaky septic tanks. The presence of chloride is from the proximity of areas that lie southward towards the Atlantic Ocean and the fact that water doesn't mix excessively at greater depths but the chloride levels are still within accepted standards for domestic use. Domestic wastes from septic tanks and improper waste disposal are the main sources of ammonium in the groundwater of the study area, and this reduces the groundwater quality in the area markedly.
\end{abstract}

Keywords: Sapele, hydro geochemical facies, contamination, ammonia, chloride

\section{Introduction}

Groundwater is the main source of freshwater in the Niger Delta, for both domestic and industrial use. The groundwater quality in the region is variable, depending on the aquifer from which it is extracted. Groundwater chemical composition is the result of the composition of water that enters the aquifers and the reactions with minerals present in the rocks forming the aquifer that may modify the composition. Groundwater, therefore, varies in composition from one well to another as a result of the varied aquifer characteristics. Within the aquifers, as groundwater flows through soil and rock particles by seepage, it dissolves substances (minerals present in the soil and rocks, contaminants such as improperly disposed industrial wastes, organic compounds, leachates from improperly disposed waste, etc.) in contact with it and becomes saturated with these dissolved substances. Dissolution of substances continues until chemical equilibrium is 
achieved between the water and the substances with which it is in contact, thereby altering the chemical composition of groundwater and hence the groundwater quality.

Land use, proximity to the coast, recharge source, soil type and resident time also influence the chemical composition of groundwater and susceptibility to pollution. In an area dominated by the petroleum industry, the quality of groundwater should be of some concern to the populace since the production of oil and gas is usually accompanied by substantial discharge of wastewater in the form of brines (composed of sodium, calcium, ammonia, sulphate, boron, chloride, trace metals and other dissolved solids) (Todd \& Mays, 2005). Brine-polluted aquifers are common place in oil producing areas due to infiltration of improperly disposed wastewaters. Areas close to the coast are susceptible to pollution by saline waters that could increase dissolved salts concentration to some $10,000 \mathrm{mg} / \mathrm{L}$ making such water unusable domestically and to some extent industrially. Recharge source, soil type and resident time are other factors that have an influence on the chemical composition of groundwater as they determine the original chemical composition of groundwater, chemical reactions in the subsurface and the amount of time (varies from days for shallow aquifers to millions of years for deep aquifers) groundwater remains within a system.

The aim of this thesis is to determine the concentrations of some major trace elements in groundwater and their spatial relationships within the study area using statistical methods.

\section{Study Area}

The $1130.61 \mathrm{~km}^{2}$-study area lies between latitude $5^{\circ} 54^{\prime} 00^{\prime \prime} \mathrm{N}$ and $5^{\circ} 24^{\prime} 00^{\prime} \mathrm{N}$, and longitude $5^{\circ} 42^{\prime} 00^{\prime \prime} \mathrm{E}$ and $5^{\circ} 54^{\prime} 00^{\prime \prime} \mathrm{E}$. The study area is located in the western part of the Niger Delta, and includes the area that covers the metropolitan city of Sapele, Warri South and Uvwie Local Government Area respectively. The area, located some 40km away from the Atlantic Ocean, has a population of over 300,000 people (Olobaniyi\&Owoyemi, 2006).

Sapele is one of the prominent commercial cities in southern Nigeria as it has a port and several oil fields and flow stations. The Warri areas of the study area are home to a port and a refinery.

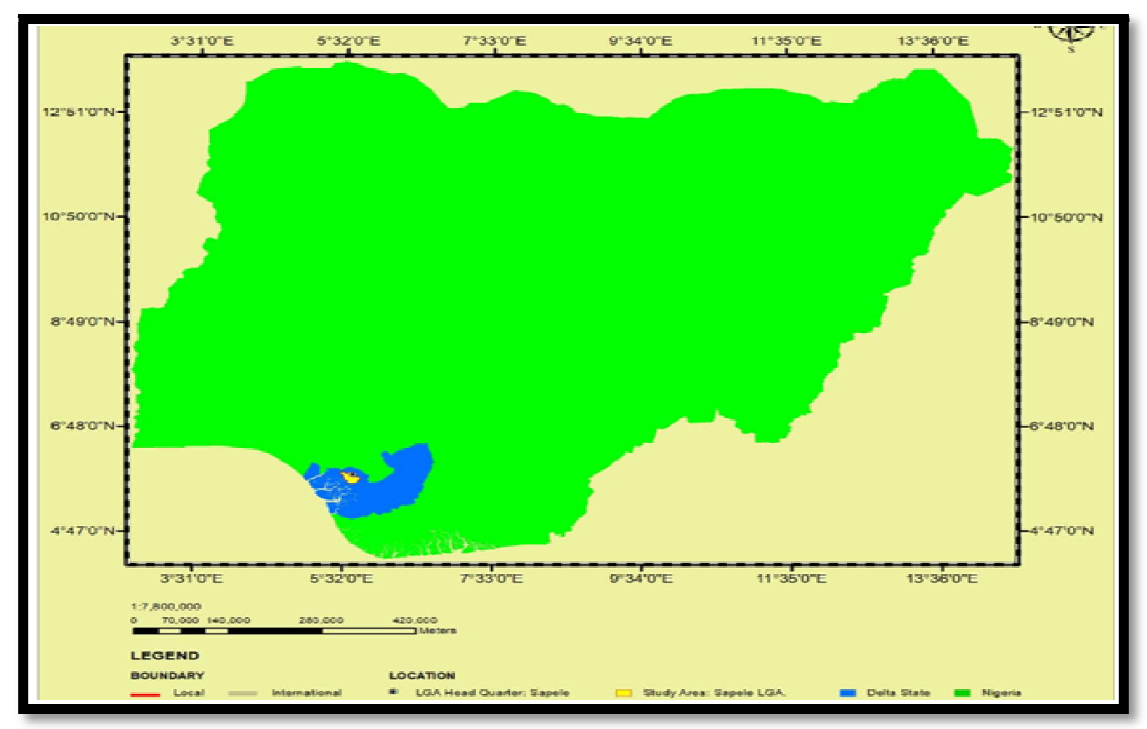

Figure 1: Ph, Total Dissolved Solids and Electrical Conductivity Data Obtained From the Field

\begin{tabular}{|c|c|c|c|c|}
\hline S/ NO & LOCATION & $\mathbf{p H}$ & TDS (ppm) & EC(mS/ cm) \\
\hline 1 & OKRD/ BH & 4.78 & 120 & 80 \\
\hline 2 & $\mathrm{CRD/BH}$ & 6.05 & 150 & 120 \\
\hline 3 & $\mathrm{UGB} / \mathrm{BH}$ & 5.17 & 220 & 360 \\
\hline 4 & $\mathrm{OGBRD/BH}$ & 3.96 & 260 & 570 \\
\hline 5 & $\mathrm{MCF} / \mathrm{BH}$ & 5.25 & 50 & 520 \\
\hline 6 & $\mathrm{ADL} / \mathrm{UBH}$ & 6.18 & 85 & 38 \\
\hline 7 & $\mathrm{ASB} / \mathrm{UBH}$ & 4.25 & 230 & 260 \\
\hline 8 & $\mathrm{UGW} / \mathrm{UBH}$ & 3.86 & 140 & 350 \\
\hline 9 & $\mathrm{URA} / \mathrm{BH}$ & 5.85 & 180 & 110 \\
\hline 10 & $\mathrm{LBRD} / \mathrm{BH}$ & 6.05 & 320 & 450 \\
\hline 11 & $0 \mathrm{KKR} / \mathrm{BH}$ & 6.38 & 300 & 420 \\
\hline 12 & $\mathrm{OGR} / \mathrm{BH}$ & 6.25 & 80 & 200 \\
\hline 13 & $\mathrm{SHRD} / \mathrm{BH}$ & 4.18 & 60 & 125 \\
\hline 14 & $\mathrm{AKNT} / \mathrm{BH}$ & 4.96 & 620 & 850 \\
\hline 15 & $\mathrm{OGB} / \mathrm{UBH}$ & 6.85 & 40 & 85 \\
\hline 16 & $\mathrm{AMK} / \mathrm{BH}$ & 6.38 & 150 & 320 \\
\hline 17 & $\mathrm{AJM} / \mathrm{BH}$ & 5.18 & 140 & 285 \\
\hline 18 & $\mathrm{EBRD} / \mathrm{BH}$ & 4.05 & 182 & 375 \\
\hline
\end{tabular}




\begin{tabular}{|c|c|c|c|c|}
\hline S/ NO & LOCATION & $\mathbf{p H}$ & TDS(ppm) & EC (mS/ cm) \\
\hline 19 & SHG/ UBH & 4.85 & 150 & 350 \\
\hline 20 & EKP/ BH & 3.96 & 50 & 120 \\
\hline 21 & OGU/ BH & 6.85 & 80 & 185 \\
\hline 22 & IYA/ UBH & 6.45 & 230 & 500 \\
\hline 23 & EST/ BH & 6.25 & 190 & 270 \\
\hline 24 & WRGRA/ BH & 5.85 & 620 & 840 \\
\hline 25 & EFGRA/ BH & 6.65 & 270 & 450 \\
\hline
\end{tabular}

Table 1: pH, Total Dissolved Solids and Electrical

Conductivity Data Obtained from the Field

\section{Results}

\begin{tabular}{|c|c|c|c|c|c|c|}
\hline BH & $\mathbf{p H}$ & TDS & EC & As & Cr & Pb \\
\hline OKRD/ BH & 4.78 & 120 & 80 & 0.01 & 0.002 & BDL \\
\hline CRD/ BH & 6.05 & 150 & 120 & BDL & 0.003 & 0.002 \\
\hline UGB/ BH & 5.17 & 220 & 360 & BDL & 0.002 & 0.001 \\
\hline OGBRD/BH & 3.96 & 260 & 570 & 0.003 & BDL & 0.004 \\
\hline MCF/ BH & 5.25 & 50 & 520 & 0.004 & 0.005 & BDL \\
\hline ADL/ UBH & 6.18 & 85 & 38 & BDL & 0.007 & BDL \\
\hline ASB/ UBH & 4.25 & 230 & 260 & BDL & 0.004 & BDL \\
\hline UGW/ UBH & 3.86 & 140 & 350 & BDL & BDL & 0.003 \\
\hline URA/ BH & 5.85 & 180 & 110 & 0.003 & 0.005 & BDL \\
\hline LBRD/ BH & 6.05 & 320 & 450 & 0.001 & 0.004 & 0.001 \\
\hline 0KR/ BH & 6.38 & 300 & 420 & 0.004 & 0.007 & 0.004 \\
\hline OGR/ BH & 6.25 & 80 & 200 & BDL & BDL & 0.003 \\
\hline SHRD/ BH & 4.18 & 60 & 125 & BDL & 0.003 & BDL \\
\hline AKNT/BH & 4.96 & 620 & 850 & 0.005 & 0.004 & BDL \\
\hline OGB/ UBH & 6.85 & 40 & 85 & 0.003 & 0.007 & 0.001 \\
\hline AMK/ BH & 6.38 & 150 & 320 & 0.001 & 0.005 & 0.003 \\
\hline AJM/ BH & 5.18 & 140 & 285 & BDL & BDL & 0.001 \\
\hline EBRD/ BH & 4.05 & 182 & 375 & BDL & BDL & BDL \\
\hline SHG/ UBH & 4.85 & 150 & 350 & 0.002 & 0.005 & BDL \\
\hline EKP/ BH & 3.96 & 50 & 120 & 0.003 & 0.004 & 0.01 \\
\hline OGU/ BH & 6.85 & 80 & 185 & BDL & 0.006 & 0.002 \\
\hline IYA/ UBH & 6.45 & 230 & 500 & 0.004 & 0.003 & 0.03 \\
\hline EST/BH & 6.25 & 190 & 270 & BDL & 0.001 & 0.002 \\
\hline WRGRA/ BH & 5.85 & 620 & 840 & BDL & 0.002 & BDL \\
\hline EFGRA/ BH & 6.65 & 270 & 450 & 0.003 & 0.007 & 0.001 \\
\hline Min. & 3.86 & 40 & 38 & 0.001 & 0.001 & 0.01 \\
\hline Max. & 6.85 & 620 & 850 & 0.01 & 0.007 & 0.004 \\
\hline Mean & 5.4596 & 196.68 & 329.32 & 0.00353846 & 0.0043 & 0.002133333 \\
\hline Median & 5.85 & 150 & - & 0.003 & 0.004 & 0.002 \\
\hline S.D. & 1.00336716 & 150.2461092 & 216.859924 & 0.00225889 & 0.001866604 & 0.001125463 \\
\hline Skewness & - & 1.829697189 & 0.91732455 & 2.01700788 & 0.052871865 & 0.397240326 \\
\hline & 0.30210829 & & & & & \\
\hline
\end{tabular}

Table 2: Statistical Representation of Physical Parameters and Trace Elements 


\begin{tabular}{|c|c|c|c|c|c|c|c|c|c|c|}
\hline$\frac{\pi}{0}$ & 0 & क̆ & 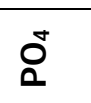 & $\hat{z}$ & है & 8 & 8 & $\sum_{2}^{2}$ & $\forall$ & 2 \\
\hline 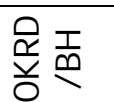 & 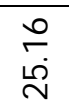 & 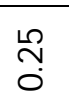 & 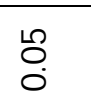 & $\stackrel{\circ}{0}_{0}^{\circ}$ & 承 & $\stackrel{8}{0} 8$ & 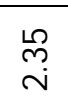 & 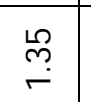 & 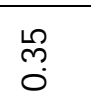 & $6 ?$ \\
\hline 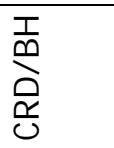 & $\begin{array}{l}\stackrel{0}{0} \\
\infty \\
\infty \\
m\end{array}$ & $\overrightarrow{\mathbb{m}_{j}}$ & $\stackrel{\overbrace{}}{\circ}$ & $\stackrel{N}{0}$ & 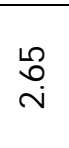 & 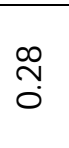 & $\stackrel{\infty}{\not}$ & $\stackrel{\stackrel{\sim}{N}}{\sim}$ & 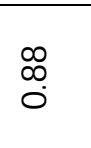 & $\dddot{\oplus}$ \\
\hline 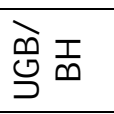 & q & 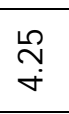 & ?. & $\overrightarrow{\ddot{g}}$ & $\begin{array}{l}\stackrel{\mathscr{L}}{0} \\
6\end{array}$ & $\begin{array}{l}\text { f? } \\
0\end{array}$ & 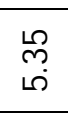 & \begin{tabular}{|l|}
$\stackrel{\sim}{n}$ \\
\end{tabular} & $\vec{m}$ & $\stackrel{\overbrace{}}{\triangle}$ \\
\hline 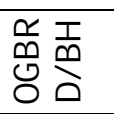 & 范 & 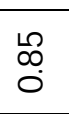 & $\stackrel{8}{0}$ & ڤ్ & 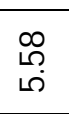 & मु. & $\begin{array}{c}\infty \\
\infty \\
\infty\end{array}$ & 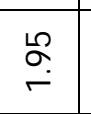 & $\underset{0}{0}$ & $\underset{\exists}{\Psi}$ \\
\hline 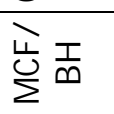 & ث艹 & 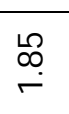 & $\overrightarrow{0}$ & $\stackrel{\infty}{\circ}$ & 获 & $\stackrel{\sigma}{0}$ & 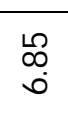 & 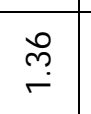 & 导 & 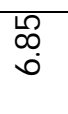 \\
\hline 窟 & $\begin{array}{l}m \\
\stackrel{m}{+} \\
\end{array}$ & 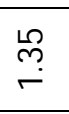 & $\stackrel{0}{\circ}$ & 今. & 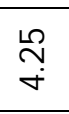 & $\stackrel{0}{0}$ & స్ & $\begin{array}{l} \\
\text { I } \\
\text { S }\end{array}$ & 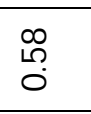 & $\underset{7}{7}$ \\
\hline 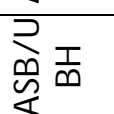 & 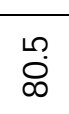 & $\stackrel{\mathscr{g}}{\rightarrow}$ & $\begin{array}{l}\text { H } \\
0\end{array}$ & $\stackrel{8}{0}$ & $\stackrel{\infty}{\vec{N}}$ & 7 & 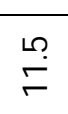 & 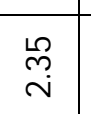 & $\begin{array}{l}\infty \\
\stackrel{\infty}{0} \\
0\end{array}$ & 范 \\
\hline 氛蒙 & m. & 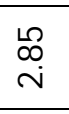 & $\begin{array}{l}0 \\
0 \\
0\end{array}$ & 9 & $\underset{m}{N}$ & $\stackrel{8}{0}$ & 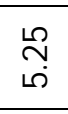 & $\begin{array}{l}\infty \\
\stackrel{\infty}{\infty} \\
\\
\end{array}$ & 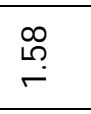 & $\mathbb{R}_{0}^{R}$ \\
\hline 蛋藏 & $\underset{\mathrm{N}}{\mathrm{N}}$ & $\stackrel{\infty}{\stackrel{\infty}{\rightarrow}}$ & 离 & $\stackrel{\infty}{\infty}$ & $\stackrel{\circ}{\circ}$ & 足 & है & 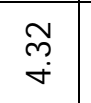 & ?잉 & 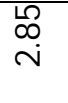 \\
\hline 甪密 & $\begin{array}{l}\overrightarrow{7} \\
: 0 \\
0\end{array}$ & लू & $\stackrel{\text { o. }}{0}$ & 号 & $\stackrel{\mathscr{N}}{\Re}$ & 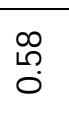 & $\underset{\tilde{ల}}{\tilde{\omega}}$ & 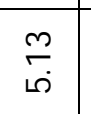 & 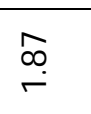 & $\infty$ \\
\hline 氞昢 & $\vec{\infty}$ & 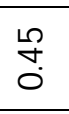 & กี่ & $\stackrel{\infty}{\infty}$ & $\begin{array}{l}0 \\
0 \\
0 \\
0\end{array}$ & $\stackrel{0}{0}$ & $\begin{array}{c}\tilde{N} \\
\tilde{+} \\
\end{array}$ & $\begin{array}{c}m \\
\vec{m} \\
\end{array}$ & $\underset{\sim}{\tilde{m}}$ & 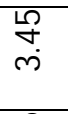 \\
\hline 居密 & $\begin{array}{l}\text { 守 } \\
\text {. }\end{array}$ & 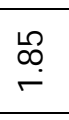 & $\begin{array}{l}\infty \\
\stackrel{\infty}{0} \\
0\end{array}$ & : & $\stackrel{\substack{\infty \\
\rightarrow}}{\rightarrow}$ & $\begin{array}{l}m \\
\stackrel{m}{0} \\
0\end{array}$ & $\begin{array}{l}\infty \\
\infty \\
\infty\end{array}$ & 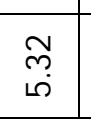 & 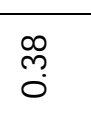 & $\begin{array}{l}\text { पूd } \\
\infty \\
\infty\end{array}$ \\
\hline 奉㩊 & $\begin{array}{l}\vec{N} \\
\text { 岕 } \\
m\end{array}$ & 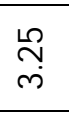 & $\stackrel{0}{0}$ & $\stackrel{0}{0}$ & $\underset{\substack{\infty\\
}}{\infty}$ & $\stackrel{2}{0}$ & $\begin{array}{l}\text { Iै } \\
\text { Ĵ }\end{array}$ & 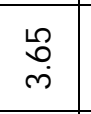 & : & đ్d \\
\hline 离䆝 & ิㅗ & $\overrightarrow{+}$ & $\stackrel{8}{\circ}$ & $\stackrel{0}{0}$ & $\stackrel{\pi}{\pi}$ & $\stackrel{\circ}{0}$ & $\begin{array}{c}m \\
m \\
\infty\end{array}$ & $\stackrel{N}{N}$ & $\begin{array}{c}m \\
\dot{m} \\
0\end{array}$ & $\stackrel{\infty}{i \overrightarrow{0}}$ \\
\hline 8 & 户े & लु & సี & $\stackrel{0}{\circ}$ & 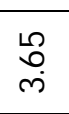 & $\stackrel{\infty}{\infty}$ & $\stackrel{M}{\sim}$ & \begin{tabular}{|c|}
$\stackrel{\infty}{\rightarrow}$ \\
$\rightarrow$
\end{tabular} & $\begin{array}{l}\infty \\
\stackrel{0}{0}\end{array}$ & $\infty$ \\
\hline 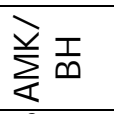 & ֻे & $\stackrel{n}{n}$ & $\stackrel{8}{\circ}$ & $\stackrel{\infty}{0}$ & $\stackrel{n}{\Lambda}$ & $\stackrel{7}{0}$ & 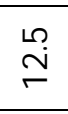 & $\vec{m}$ & 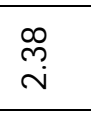 & 量 \\
\hline$\sum_{4}$ & 呙 & $\stackrel{m}{\stackrel{m}{\infty}}$ & $\stackrel{\text { ने }}{0}$ & ఫ్ & 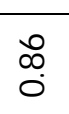 & $\stackrel{n}{0}$ & $\stackrel{\hat{m}}{\hat{b}}$ & $\mid \begin{array}{c}\stackrel{\leftrightarrow}{\circ} \\
\stackrel{\sim}{+}\end{array}$ & $\underset{\sim}{\tilde{\oplus}}$ & 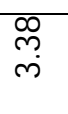 \\
\hline 商票 & $\begin{array}{l}\text { Nิ } \\
\text { Oे }\end{array}$ & $\begin{array}{c}5 \\
\\
+\end{array}$ & $\stackrel{10}{0}$ & $\stackrel{7}{0}$ & 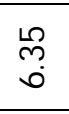 & $\stackrel{\infty}{0}$ & 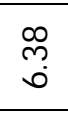 & 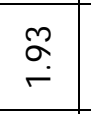 & $\stackrel{\substack{p \\
\sim}}{\sim}$ & 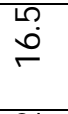 \\
\hline 焉壱 & 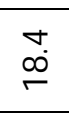 & సै & $\stackrel{0}{0}$ & $\stackrel{8}{\circ}$ & $\begin{array}{l}\stackrel{\mathscr{R}}{0} \\
\stackrel{0}{0}\end{array}$ & 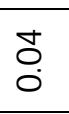 & $\vec{N}$ & $\stackrel{\stackrel{\leftrightarrow}{n}}{\text { N }}$ & $\stackrel{\infty}{\circ}$ & ถู่ \\
\hline 商出 & $\overrightarrow{9}$ & $\stackrel{n}{\circ}$ & $\tilde{O}$ & 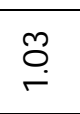 & : & : & $\begin{array}{l}\infty \\
\stackrel{m}{\infty} \\
\dot{m}\end{array}$ & 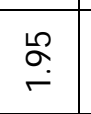 & $\begin{array}{l}\text { 哭 } \\
\text { o }\end{array}$ & $\stackrel{\text { İ }}{\mathrm{I}}$ \\
\hline 害苗 & ผู่ & $\stackrel{\infty}{\rightarrow}$ & $\stackrel{\circ}{\circ}$ & $\stackrel{n}{0}$ & $\stackrel{\leftrightarrow}{\stackrel{\leftrightarrow}{\leftrightarrow}}$ & $\stackrel{\circ}{\circ}$ & $\underset{\vec{f}}{\overrightarrow{+}}$ & 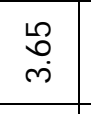 & 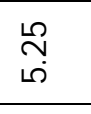 & 岕 \\
\hline 发密 & $\stackrel{N}{N}$ & $\stackrel{9}{0}$ & $\begin{array}{l}\stackrel{\infty}{0} \\
\stackrel{0}{0}\end{array}$ & $\stackrel{\infty}{0}$ & $\stackrel{\mathscr{m}}{\mathscr{m}}$ & $\stackrel{\sigma}{\circ}$ & $\begin{array}{l}\qquad \\
0 \\
0 \\
0\end{array}$ & 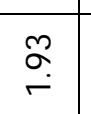 & $\underset{\sim}{\tilde{m}}$ & $\begin{array}{c}\hat{1} \\
\infty \\
\infty \\
\infty\end{array}$ \\
\hline 昜州 & $\begin{array}{l}0 . \\
\dot{m} \\
\end{array}$ & $\mathscr{D}_{0}^{\circ}$ & m. & $\stackrel{\infty}{\circ}$ & 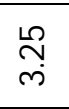 & $\stackrel{n}{0}$ & 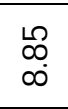 & \begin{tabular}{|l|l}
$\stackrel{u}{ }$ \\
\end{tabular} & $\begin{array}{l}\infty \\
\stackrel{\infty}{0}\end{array}$ & 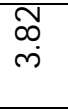 \\
\hline
\end{tabular}




\begin{tabular}{|c|c|c|c|c|c|c|c|c|c|c|}
\hline 部 & ठ & ஜ" & 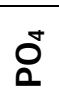 & $\overbrace{}^{\infty}$ & $\bar{Z}$ & 8 & है & $\sum$ & $\mathscr{Z}$ & 2 \\
\hline$\sum_{z}^{\infty}$ & $\stackrel{m}{\sigma}$ & $\begin{array}{l}\infty \\
\infty \\
0 \\
0\end{array}$ & $\stackrel{10}{\sim}$ & $\stackrel{\text { L̊ }}{\circ}$ & $\begin{array}{l}\vec{\infty} \\
\dot{10}\end{array}$ & $\stackrel{\hat{0}}{0}$ & $\stackrel{N}{\stackrel{N}{\circ}}$ & 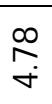 & $\stackrel{+}{\underset{-}{-}}$ & $\stackrel{m}{m}$ \\
\hline 商衣 & $\begin{array}{l}+1 \\
\infty\end{array}$ & $\stackrel{+}{\rightarrow}$ & $\ddot{\circ}$ & ָָ & $\stackrel{\varphi}{\rightarrow}$ & $\hat{o}$ & $\underset{\infty}{m}$ & $\dddot{M}_{0}$ & $\stackrel{\bullet}{\sim}$ & mु \\
\hline$\sum$ & $\begin{array}{c}\hat{m} \\
\infty \\
\infty\end{array}$ & $\stackrel{9}{0}$ & $\stackrel{-1}{0}$ & \%) & $\begin{array}{l}10 \\
0 \\
0\end{array}$ & $\ddot{0}$ & $\stackrel{\stackrel{\leftrightarrow}{\oplus}}{\text { ì }}$ & $\stackrel{\leftrightarrow}{\text { m }}$ & $\stackrel{\infty}{0}$ & ن \\
\hline$\sum^{\frac{x}{4}}$ & ஜே! & $\stackrel{\stackrel{\leftrightarrow}{\rightarrow}}{\rightarrow}$ & $\stackrel{0}{\circ}$ & 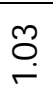 & $\stackrel{\stackrel{N}{N}}{N}$ & $\stackrel{p}{0}$ & $\stackrel{m}{\stackrel{n}{\sim}}$ & लै & $\begin{array}{l}\text { مึ } \\
\text { เึ }\end{array}$ & f. \\
\hline$\frac{5}{\sum^{8}}$ & $\begin{array}{l}\text { Oे } \\
\text { M. } \\
\dot{m}\end{array}$ & $\begin{array}{l}0 \\
\text { ్ֶ } \\
\text { o } \\
-1\end{array}$ & $\begin{array}{l}0 \\
\stackrel{2}{2} \\
0 \\
0\end{array}$ & 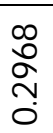 & $\begin{array}{l}\infty \\
\infty \\
\infty \\
m \\
\text { m. } \\
\end{array}$ & 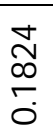 & 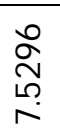 & 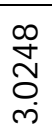 & 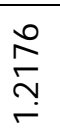 & $\underset{\infty}{m}$ \\
\hline $\begin{array}{l}5 \\
\sum_{2}^{6}\end{array}$ & $\begin{array}{l}m \\
\stackrel{\infty}{N}\end{array}$ & & $\hat{\circ}$ & $\stackrel{0}{\stackrel{1}{0}}$ & $\begin{array}{l}\stackrel{\varphi}{\emptyset} \\
\stackrel{\leftrightarrow}{i}\end{array}$ & $\underset{\sigma}{-1}$ & ڤึ & 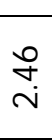 & $\begin{array}{l}\infty \\
\infty \\
0\end{array}$ & 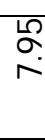 \\
\hline के & 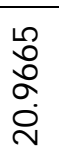 & 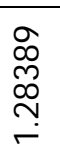 & $\begin{array}{l}\hat{0} \\
0 \\
0 \\
0 \\
0 \\
0\end{array}$ & 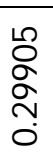 & 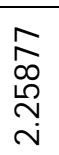 & 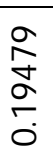 & $\begin{array}{l}\vec{b} \\
\stackrel{\omega}{m} \\
\stackrel{n}{0}\end{array}$ & 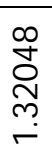 & $\begin{array}{l}0 \\
\stackrel{0}{0} \\
\stackrel{0}{0} \\
\end{array}$ & 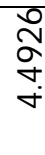 \\
\hline $\begin{array}{l}\text { क्ष } \\
\text { है } \\
\text { है } \\
\text { है }\end{array}$ & 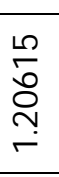 & $\begin{array}{l}0 \\
\stackrel{1}{1} \\
0 \\
0 \\
0 \\
0\end{array}$ & $\begin{array}{l}\tilde{\sigma} \\
\overrightarrow{0} \\
\stackrel{0}{ } \\
0\end{array}$ & 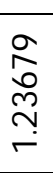 & 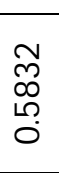 & 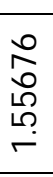 & 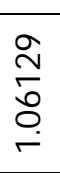 & 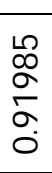 & $\begin{array}{l}\infty \\
\infty \\
\stackrel{\infty}{\infty} \\
\stackrel{\infty}{\sim} \\
\stackrel{\sim}{-1}\end{array}$ & $\begin{array}{l}8 \\
0 \\
8 \\
0\end{array}$ \\
\hline
\end{tabular}

Table 3: Statistical Representation of Anions and Cations

The results of the raw laboratory analysis are presented in Table 3. These results show that most of the sample locations have alarmingly poor water quality. Most of the samples obtained from both cased and uncased boreholes have $\mathrm{pH}$ values of less than 6.50 (less than the Nigerian Standard for Drinking water Quality (NSDWQ,2007) and the United States Environmental Protection Agency (EPA, 2012) standards for drinking water) and are therefore acidic. Theboreholes in Akintola, sapele and Warri GRA have TDS values of $620 \mathrm{mg} / \mathrm{l}$ which is above the Nigerian Standard for Drinking water Quality (NSDWQ,2007) and the United States Environmental Protection Agency (EPA, 2012) of $500 \mathrm{mg} / \mathrm{l..The}$ analysis of arsenic, chromium and lead show that their concentrations fall below the Nigerian Standard for Drinking water Quality (NSDWQ,2007) and the United States Environmental Protection Agency (EPA, 2012) standards for drinking water which are $0.01 \mathrm{mg} / \mathrm{l}, 0.05 \mathrm{mg} / \mathrm{l}$ and $0.01 \mathrm{mg} / \mathrm{l}$ respectively.EuropeanUnionDrinkingWaterDirective(1998)forammonium in drinking water is $0.5 \mathrm{mg} / \mathrm{l}$, the mean value for ammonium is $3.44 \mathrm{mg} / \mathrm{l}$ for all sampled boreholes which is well above the EU(EU,1998)standards

\section{Summarized Statistics of Groundwater Physical and Chemical Characteristics}

\begin{tabular}{|c|c|c|c|c|c|c|c|}
\hline & Min. & Max. & Mean & Median & US EPA (2012) & EU DWD (1998) & NSDWQ(2007) \\
\hline $\mathrm{pH}$ & 3.86 & 6.85 & 5.4596 & 5.85 & $6.5-8.5$ & & $6.5-8.5$ \\
\hline $\mathrm{TDS}$ & 40 & 620 & 196.68 & 150 & 500 & & 500 \\
\hline $\mathrm{EC}$ & 38 & 650 & 329.32 & 320 & & & 1000 \\
\hline $\mathrm{As}$ & 0.001 & 0.01 & 0.00354 & 0.003 & & & 0.01 \\
\hline $\mathrm{Cr}$ & 0.001 & 0.007 & 0.0043 & 0.004 & & & 0.05 \\
\hline $\mathrm{Pb}$ & 0.001 & 0.004 & 0.00213 & 0.002 & & & 0.01 \\
\hline $\mathrm{Cl}$ & 8.37 & 90.38 & 36.334 & 28.25 & 250 & & 250 \\
\hline $\mathrm{SO}_{4}$ & 0.19 & 4.25 & 1.9636 & 1.85 & & 250 & 100 \\
\hline $\mathrm{PO}_{4}$ & 0.01 & 0.19 & 0.0956 & 0.07 & & & 5 \\
\hline $\mathrm{NO}_{3}$ & 0.03 & 1.03 & 0.2968 & 0.16 & 10 & 0.5 & 50 \\
\hline $\mathrm{NH}_{4}$ & 0.75 & 7.25 & 3.3488 & 2.65 & & & \\
\hline $\mathrm{HCO}_{3}$ & 0.01 & 0.73 & 0.1824 & 0.11 & & 200 & \\
\hline $\mathrm{Ca}$ & 2.35 & 17.25 & 7.5296 & 6.95 & & 200 & \\
\hline $\mathrm{Mg}$ & 1.35 & 6.32 & 3.0248 & 2.46 & & & \\
\hline $\mathrm{K}$ & 0.08 & 5.25 & 1.2176 & 0.88 & & 200 & 200 \\
\hline $\mathrm{Na}$ & 1.85 & 17.35 & 8.33 & 7.95 & & & \\
\hline
\end{tabular}

Table 4: Compared against US EPA (EPA, 2012), EU DWD (EU, 1998) and NSDWQ 


\begin{tabular}{|c|c|c|}
\hline Degree of Hardness & Grains per Gallon (gpg) & $\begin{array}{c}\text { Parts per Million (ppm) } \\
\mathbf{1} \mathbf{~ g p g = 1 7 . 1 ~ p p m}\end{array}$ \\
\hline Soft & $<1.0$ & $<17.0$ \\
\hline Slightly Hard & $1.0-3.5$ & $17.1-60$ \\
\hline Moderately Hard & $3.5-7.0$ & $60-120$ \\
\hline Hard & $7.0-10.5$ & $120-180$ \\
\hline Very Hard & 10.5 & $>180$ \\
\hline
\end{tabular}

Table 5: Showing Standards for Hardness in Water

Source: American Society of Agricultural Engineers (S-339) and the Water Quality Association (WQA)

Tables show that the study area has very high mean TDS concentration. Using the equation to determine the hardness of water in the study area, we classified the water of the study area as very hard with mean hardness of 11.5. Akintola and Warri GRA boreholes have the highest hardness value of 47.

4.1. Correlation Matrix

\begin{tabular}{|c|c|c|c|c|c|c|c|c|}
\hline & $\mathbf{P h}$ & TDS & $\mathbf{E C}$ & $\mathbf{A s}$ & $\mathbf{C r}$ & $\mathbf{P b}$ & $\mathbf{C l}$ & $\mathbf{S O}_{4}$ \\
\hline $\mathrm{pH}$ & 1 & 0.204511 & -0.01686 & 0.11112 & 0.024385 & 0.083518 & 0.275941 & 0.083518 \\
\hline $\mathrm{TDS}$ & 0.19988 & 1 & -0.09913 & -0.29664 & -0.01979 & 0.085964 & 0.127732 & -0.10783 \\
\hline $\mathrm{EC}$ & 0.210617 & 0.403846 & 1 & 0.181466 & -0.20233 & 0.028296 & 0.279037 & 0.011487 \\
\hline $\mathrm{As}$ & -0.23805 & -0.23805 & -0.0347 & 1 & -0.29664 & 0.081026 & 0.204511 & -0.287 \\
\hline $\mathrm{Cr}$ & -0.26628 & 0.838278 & -0.03887 & 0.12794 & 1 & -0.04716 & 0.20048 & -0.25852 \\
\hline $\mathrm{Pb}$ & 0.408016 & -0.12756 & -0.2954 & -0.01998 & 0.133479 & 1 & 0.043486 & 0.403846 \\
\hline $\mathrm{Cl}$ & -0.29539 & 0.41472 & 0.408016 & -0.17602 & 0.046608 & 0.181466 & 1 & -0.38871 \\
\hline $\mathrm{SO}_{4}$ & -0.31922 & -0.00332 & -0.01683 & -0.10467 & 0.16816 & -0.13938 & 0.053799 & 1 \\
\hline $\mathrm{PO}_{4}$ & -0.16089 & 0.134216 & 0.11112 & -0.13189 & -0.05042 & 0.135465 & 0.061927 & 0.108432 \\
\hline $\mathrm{NO}_{3}$ & 0.18149 & 0.559458 & -0.07488 & -0.18708 & 0.021437 & -0.1948 & 0.275941 & -0.11144 \\
\hline $\mathrm{NH}_{4}$ & 0.022411 & 0.232008 & -0.02936 & 0.027155 & 0.134216 & -0.14941 & 0.5588 & -0.09844 \\
\hline $\mathrm{HCO}$ & -0.23777 & 0.204044 & -0.10113 & 0.163411 & -0.29236 & 0.014014 & 0.284765 & 0.5588 \\
\hline $\mathrm{Ca}$ & -0.13189 & 0.022411 & 0.30699 & -0.17733 & 0.133476 & 0.559458 & -0.1509 & 0.064799 \\
\hline $\mathrm{Mg}$ & -0.01979 & -0.04381 & 0.148752 & 0.210201 & -0.29236 & -0.10113 & -0.42931 & -0.11679 \\
\hline $\mathrm{K}$ & 0.139396 & 0.309058 & -0.10415 & 0.053799 & -0.25322 & 0.210201 & -0.01998 & -0.03327 \\
\hline $\mathrm{Na}$ & 0.635746 & -0.20233 & 0.41472 & -0.37184 & 0.061927 & 0.224846 & 0.121394 & -0.2954 \\
\hline
\end{tabular}

Table 6: Correlation Table Showing Relationship between Analyzed Parameters

\begin{tabular}{|c|c|c|c|c|c|c|c|}
\hline $\mathbf{P O}_{\mathbf{4}}$ & $\mathbf{N O}_{\mathbf{3}}$ & $\mathbf{N H}_{\mathbf{4}}$ & $\mathbf{H C O}$ & $\mathbf{C a}$ & $\mathbf{M g}$ & $\mathbf{K}$ & $\mathbf{N a}$ \\
\hline 0.056242 & 0.12564 & 0.034267 & 0.034562 & 0.054637 & 0.045367 & 0.346789 & 0.045637 \\
\hline-0.05042 & -0.08765 & -0.01526 & -0.10236 & -0.03458 & -0.03456 & -0.03453 & -0.03453 \\
\hline-0.14941 & 0.23459 & 0.034522 & 0.165637 & 0.034678 & 0.325678 & 0.034556 & 0.153663 \\
\hline 0.02225 & 0.13865 & 0.016753 & -0.26789 & -0.03246 & -0.03458 & -0.02458 & -0.02458 \\
\hline 0.064799 & 0.034672 & 0.034568 & 0.236788 & 0.020345 & 0.023443 & 0.126746 & 0.023566 \\
\hline 0.234304 & -0.02548 & -0.03452 & -0.02355 & -0.26759 & 0.043467 & 0.34629 & -0.02358 \\
\hline 0.061222 & -0.02562 & -0.01256 & 0.341267 & 0.045699 & -0.03453 & -0.02438 & 0.346798 \\
\hline-0.33307 & -0.04533 & 0.01654 & -0.03457 & -0.03426 & -0.03426 & -0.02675 & -0.02369 \\
\hline 1 & 0.25639 & 0.235478 & 0.126788 & 0.235678 & 0.65378 & 0.564789 & 0.563875 \\
\hline-0.10467 & 1 & -0.32675 & -0.02564 & -0.03453 & -0.03456 & -0.43528 & -0.23417 \\
\hline 0.300716 & 0.023347 & 1 & 0.034526 & 0.045327 & 0.023689 & 0.034527 & -0.32418 \\
\hline-0.13256 & 0.023578 & 0.234577 & 1 & -0.02346 & -0.03426 & -0.03453 & -0.15339 \\
\hline 0.279037 & -0.03545 & -0.21377 & -0.02346 & 1 & -0.03412 & -0.02675 & 0.563452 \\
\hline-0.25852 & -0.0654 & -0.23415 & -0.03453 & -0.09368 & 1 & -0.02347 & -0.01568 \\
\hline 0.234304 & 0.034627 & 0.023478 & 0.053676 & -0.05345 & 0.025678 & 1 & 0.215786 \\
\hline-0.26579 & 0.143678 & -0.02347 & -0.04355 & -0.02347 & 0.227834 & 0.156473 & 1 \\
\hline
\end{tabular}

Table 7: Correlation Matrix Continued 
Pearson's correlation matrix shows the relationship between the parameters .Only correlation coefficients above 0.5 were chosen since these indicate very highpositive correlation.

\subsection{Hierarchical Cluster Analyses Results and Stiff Plots}

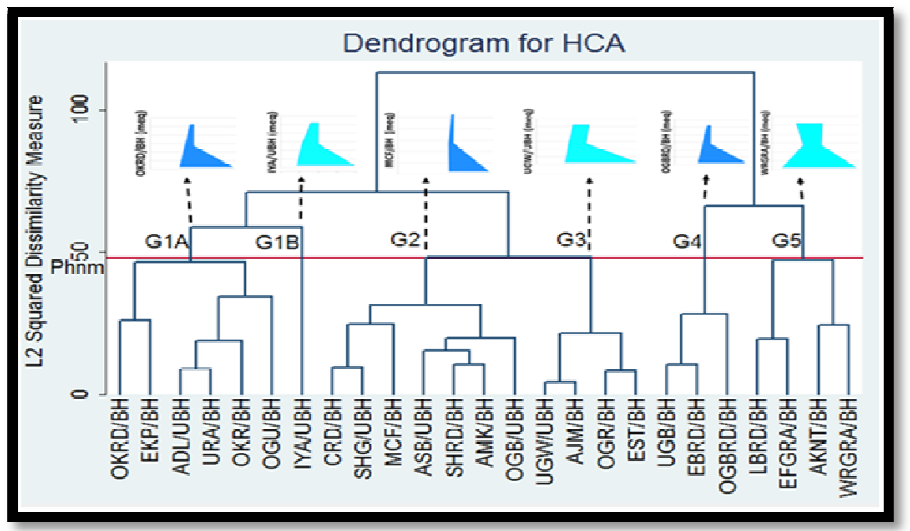

Figure 2: Cluster Analyses Dendogram Correlated with Stiff Diagrams of Sample Locations

The classification of the samples into clusters is based on a visual observation of the dendogram. Chloride is the most dominant constituent in this case and has the highest linkage distance.

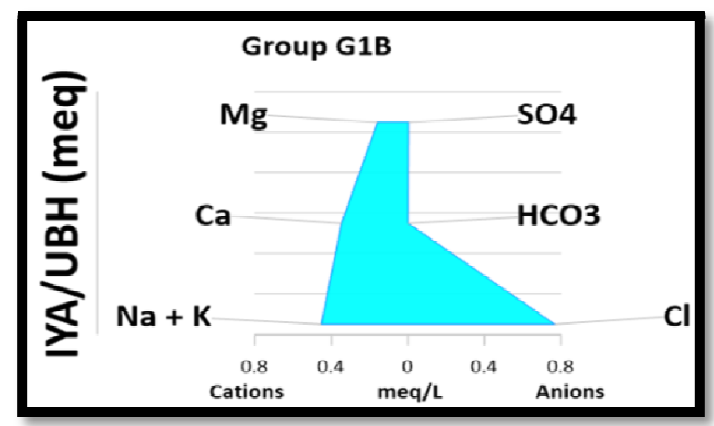

Figure 3: Stiff Diagram Indicating Parameters Used for Plotting

4.3. Principal Component Analyses Results

\begin{tabular}{|c|c|c|c|c|}
\hline Component & Eigenvalue & Difference & Proportion & Cumulative \\
\hline Comp1 & 2.46293 & 0.606188 & 0.1539 & 0.1539 \\
\hline Comp2 & 1.85674 & 0.0465308 & 0.116 & 0.27 \\
\hline Comp3 & 1.81021 & 0.0296736 & 0.1131 & 0.3831 \\
\hline Comp4 & 1.78054 & 0.0229129 & 0.1113 & 0.4944 \\
\hline Comp5 & 1.75762 & 0.181181 & 0.1099 & 0.6043 \\
\hline Comp6 & 1.57644 & 0.130455 & 0.0985 & 0.7028 \\
\hline Comp7 & 1.44599 &. & 0.0904 & 0.7932 \\
\hline
\end{tabular}

Table 8: Principal Components with Varimax Applied

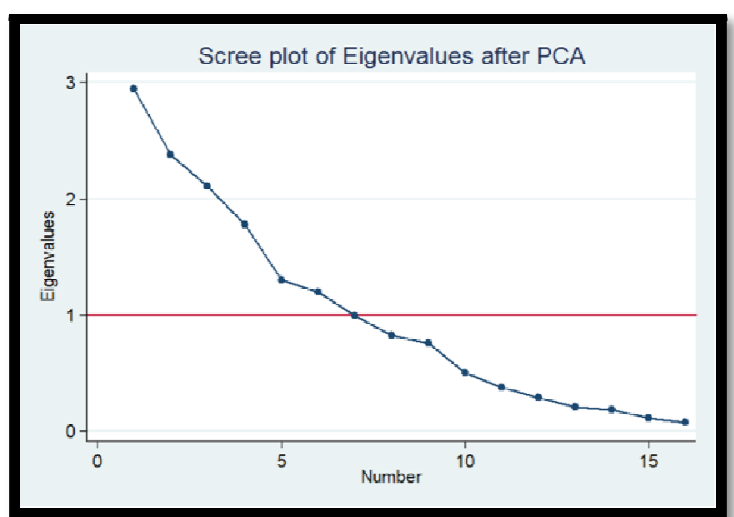

Figure 4: Scree Plot for Eigenvalues after Principal Component Analysis 
From the graph, only Eigen values above 1 were retained which shows that seven (7) factors are responsible for the variation in hydro geochemistry of the samples.

\begin{tabular}{|l|c|c|c|c|c|c|c|c|}
\hline Variable & Comp1 & Comp2 & Comp3 & Comp4 & Comp5 & Comp6 & Comp7 & Unexplained \\
\hline $\mathrm{pH}$ & & 0.4143 & & & & 0.3205 & & 0.1869 \\
\hline $\mathrm{TDS}$ & 0.5881 & & & & & & & 0.08911 \\
\hline $\mathrm{EC}$ & 0.5445 & & & & & & & 0.1951 \\
\hline $\mathrm{Ca}$ & & 0.6036 & & & & & & 0.2498 \\
\hline $\mathrm{Mg}$ & & & 0.4838 & & & & -0.3497 & 0.132 \\
\hline $\mathrm{K}$ & & & & & & 0.7125 & & 0.2075 \\
\hline $\mathrm{Na}$ & & -0.4405 & & & & & & 0.321 \\
\hline $\mathrm{Cl}$ & & & & & & & 0.8072 & 0.09337 \\
\hline $\mathrm{SO4}$ & & & & & 0.6984 & & & 0.1834 \\
\hline $\mathrm{HCO3}$ & & & 0.6756 & & & & & 0.2134 \\
\hline $\mathrm{NO3}$ & & & -0.3539 & 0.3754 & & & & 0.4003 \\
\hline PO4 & & & & -0.6554 & & & & 0.1583 \\
\hline $\mathrm{NH4}$ & 0.5287 & & & & & & & 0.2154 \\
\hline As & & & & & -0.4638 & & & 0.3037 \\
\hline $\mathrm{Cr}$ & & 0.3779 & & 0.5449 & & & & 0.1181 \\
\hline $\mathrm{Pb}$ & & & & & -0.4025 & 0.3529 & & 0.2421 \\
\hline & & & & & & & & \\
\hline Proportion (9) & 15.39 & 11.6 & 11.31 & 11.13 & 10.99 & 9.85 & 9.04 & \\
\hline Cumulative (919 & 15.39 & 27 & 38.31 & 49.44 & 60.43 & 70.28 & 79.32 & \\
\hline
\end{tabular}

Table 9: Rotated Component Loadings (Pattern Matrix) and Unique Variances

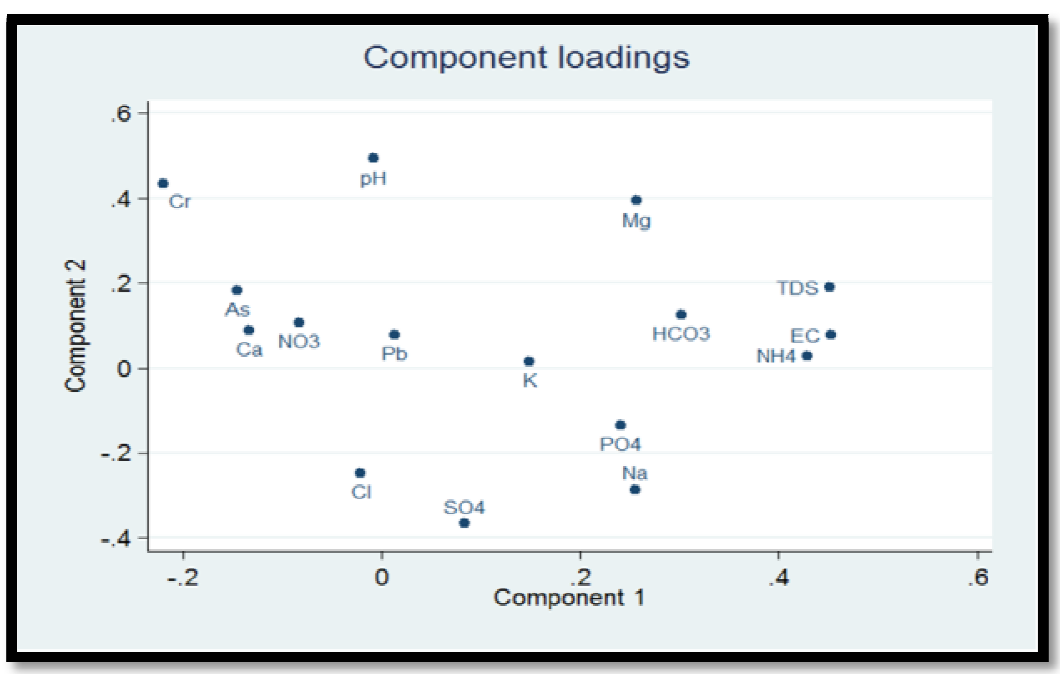

Figure 5: Graph of Component Loadings for Components 1 and 2 Showing Parameters Loading on Components

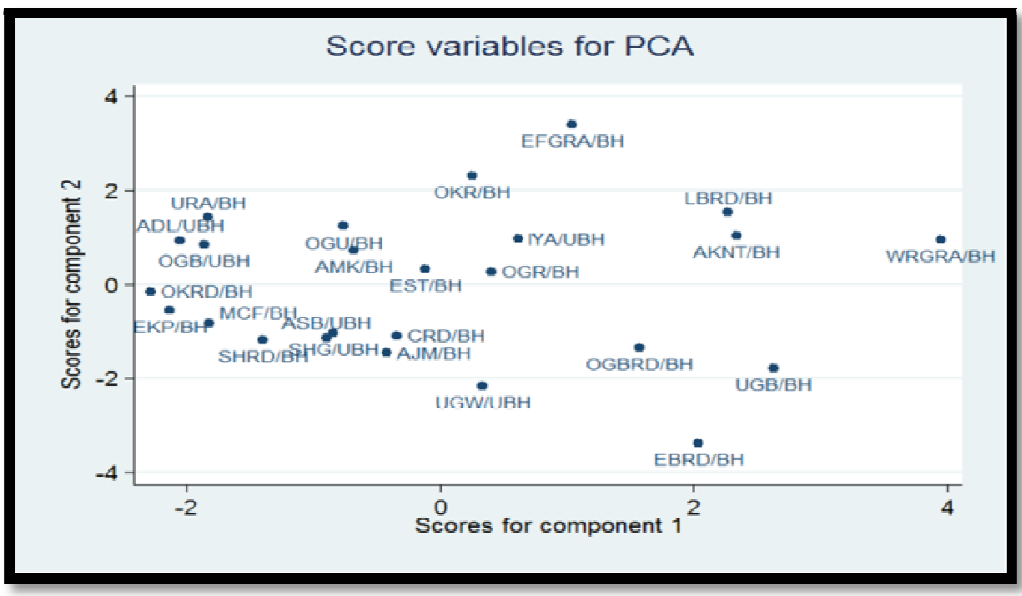

Figure 6: Graph of Score Variables For Component 1 And 2 Showing Sample Locations Loading On Components

Score variables for the first two factors plotted against each other. The Warri GRA borehole is heavily linked to the first factor. The Shell road borehole is the least linked with factor 1. Labored road, Akintola and Effurun GRA are the most linked with factor 2. 


\subsection{Piper Diagram}

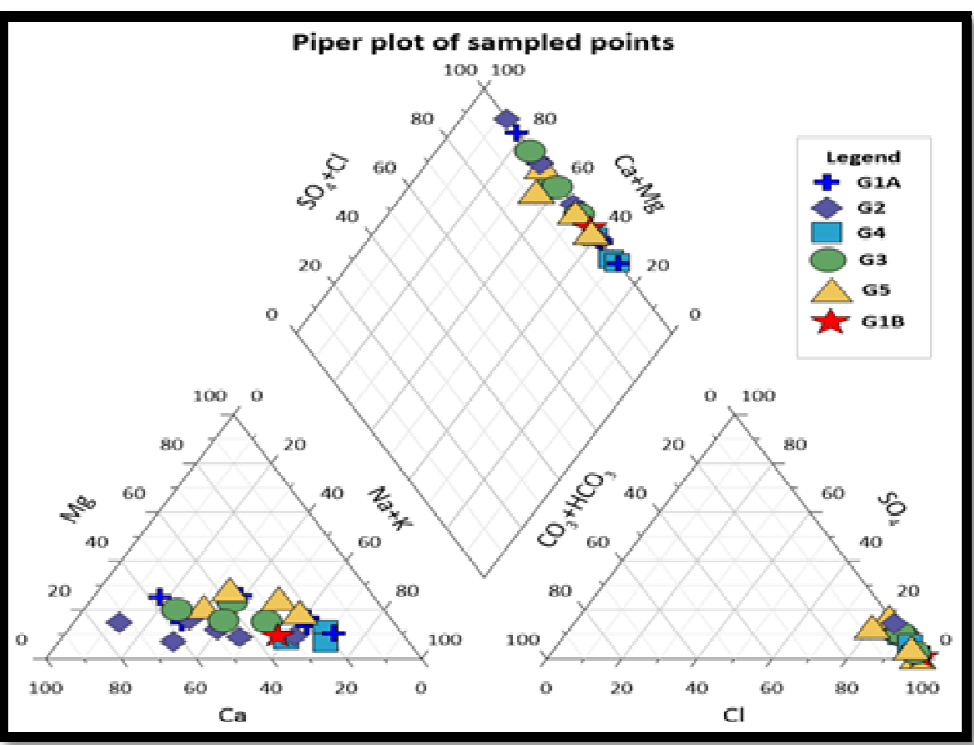

Figure 7: Piper Plot for Study Area

The Piper plot shows that the all the samples belong in the $\mathrm{Ca}^{2+-} \mathrm{Mg}^{2+-} \mathrm{Cl}-\mathrm{SO}_{4}{ }^{2-}$ hydrochemical facies. Calcium ion is the dominant parameter both in the anions and cations in the study area. The waters of the study area can therefore be classified as a $\mathrm{Ca}^{2+} \mathrm{Cl}^{-}$waters.

\subsection{Durov Plot}

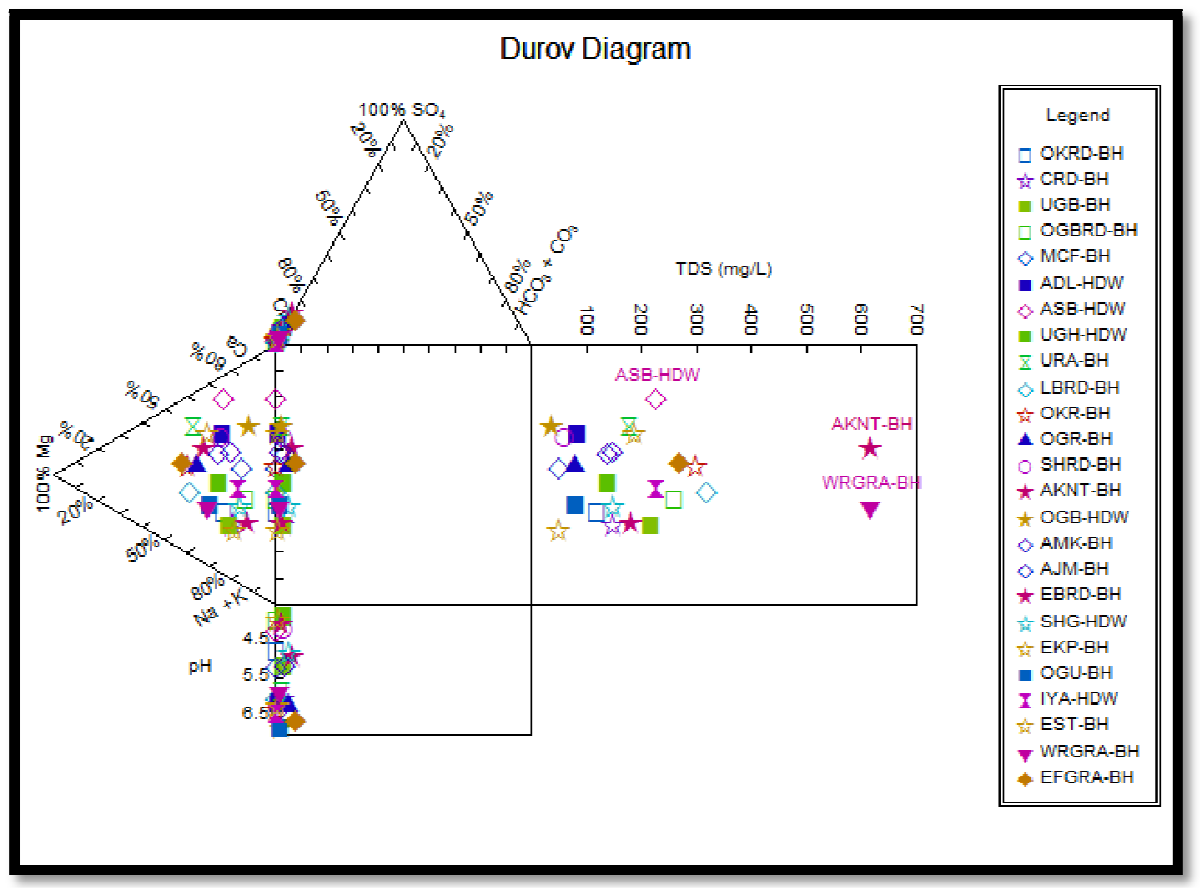

Figure 8: Durov Plot of the Study Area

ThDurov plot shows the relationship between physical parameters such as pH,EC and TDS with chemical parameters which include trace metals, anions and cations. The plot shows that areas with high TDS values equally have high concentration of $\mathrm{Na}$, $\mathrm{Ca}$ and Mg.

\subsection{Schoeller Diagram}

The Schoeller diagram gives a graphical representation of the average composition of the major cations and anions present in the water samples of the study area. Warri GRA, Eboh road Akintola,Ogunu and Effurun GRA boreholes have the highest chloride concentrations which implies a susceptibility to saline intrusion and hence not suitable for drinking. 


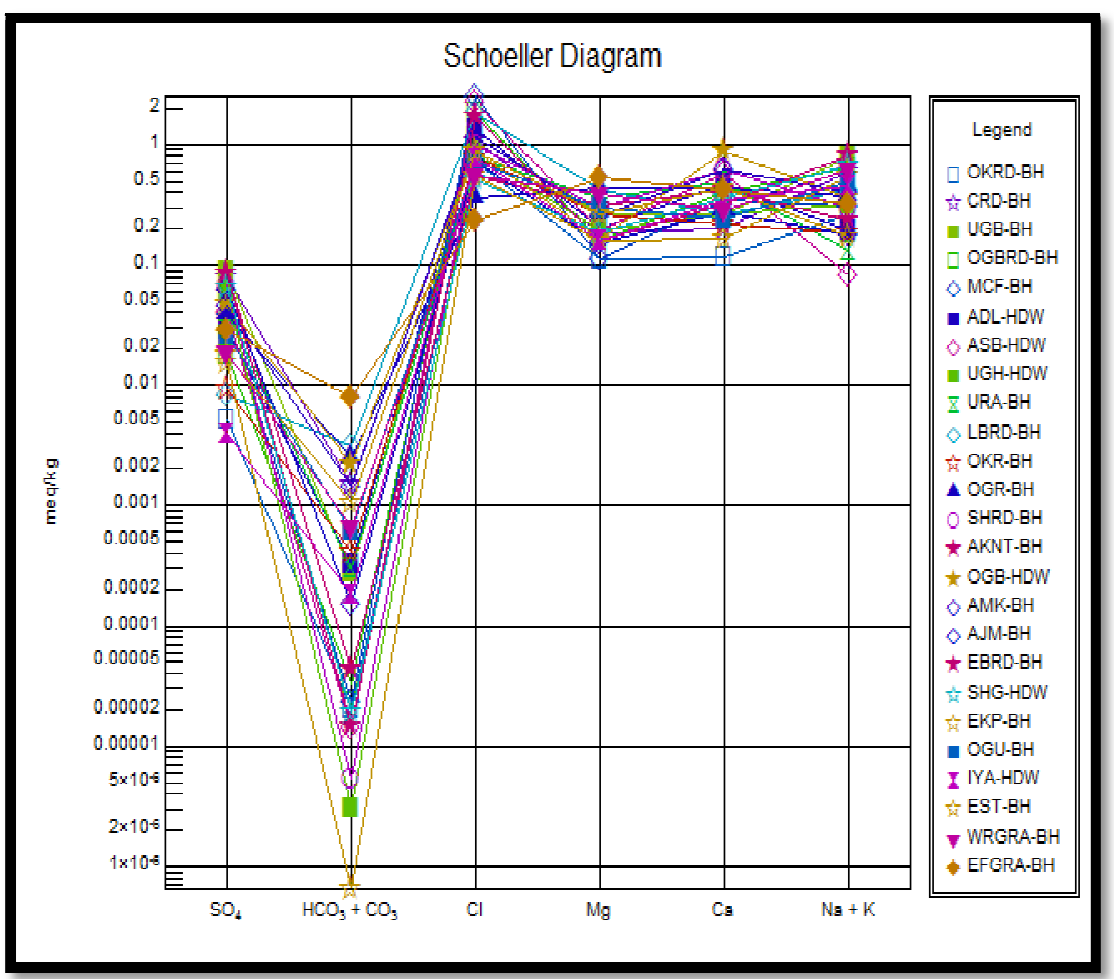

Figure 9: Schoeller Diagram of Study Area

\section{Conclusion}

The groundwater within the study area is mostly acidic with high chloride concentrations. Contamination by anthropogenic sources is mainly as a result of waste waters in the form of brines released as by-products from oil exploration activities and also from leaky septic tanks as can be seen by the relatively high concentration of chloride and ammonia. The presence of chloride is from the proximity of areas that lie south ward towards the Atlantic Oceanand the fact that water doesn't mix excessively at greater depths but the chloride levels are still within accepted standards for domestic use. Domestic wastes from septic tanks and improper waste disposal are the main sources of ammonium in the groundwater of the study area, and this reduces the groundwater quality in the area markedly.

HCA and PCA are very reliable statistical methods for water analysis in general. The graphical methods are essential in providing a visual guide to access dominant chemical constituents.

The following findings were made:

- Chloride and calcium ions are the dominant ions and they have anthropogenic contribution. The groundwaters of the study area are thus classified in the $\mathrm{Ca}-\mathrm{Cl}$ hydrogeochemical facies.

- The shallower uncased boreholes have a lower chloride concentration compared with the deeper cased boreholes; this is explained by the depth. (Todd and Mays, 2005).

- Using GPS readings and other hydrogeological parameters, we concluded for a SW trend for the direction of flow of groundwater for the study areas.

- The level of ammonium and nitrate ions at higher than geogenic (resulting from geologic processes) levels are an important indicator of pollution from sewage. Cement mortar used for coating the water well may also release considerable amounts of ammonia into drinking-water and compromise disinfection with chlorine.

- $\mathrm{As}, \mathrm{Cr}$ and $\mathrm{Pb}$ show very strong positive correlation and their presence in the groundwater is most probably as a result of anthropogenic activities.

- The very high hardness of the ground waters in the study area is most probably as a result of equally high calcium and chloride concentrations.

\section{References}

i. Adejuwon, O. A., 2012. Rainfall Seasonality in the Niger Delta Belt, Nigeria. Journal of Geography and Regional Planning, 5(2), pp. 51-60.

ii. Akpoborie, I. A., Aweto, K. E. \& Oghenero, O., 2014. Urbanization and Major Ion Hydrogeochemistry of the ShallowAquifer at the Effurun - Warri Metropolis, Nigeria. Environment and Pollution, 4(1), pp. 37-43.

iii. Akpoborie, I. A., Etobro A.A.I., Nfor, B. and Odagwe, S. (2011). Aspects of the geology and groundwater conditions of Asaba, Nigeria. Scholars Research Library Archives of Applied Science Research, Vol. 3 (2): 537-550

iv. EU, 1998. Guidance document on reporting under the Drinking Water Directive 98/ 83/ EC. Official Journal of the European Communities, Volume L 330, pp. 35-47.

v. Ezemonye, L.I and Enuneku, A.A. (2012). Hepatic bioaccumulation of cadmium in chorded bull frog, Hoplobatrachusb occipitals and flat backed toad, Bufo maculates. International Journal of Aquatic Science. ISSN; 2008-8019, vol 3, (1): 15-22. 
vi. Güler, C. \& Thyne, G. D., 2004. Hydrologic and geologic factors controlling surface and groundwater chemistry in Indian Wells-Owens Valley area, southeastern California, USA. Journal of Hydrology,Volume 285, pp. 177-198.

vii. Güler, C., Thyne, G., McCray, J. E. \& Turner, A. K., 2002. Evaluation and graphical and multivariate statistical methods for classification of water chemistry data. Hydrogeology, 2002(10), pp. 455-474.

viii. Hu, Y., Liu, X, Bai, J., Shih, K., Zeng, E.Y. and Cheng, H. (2013). Assessing heavy metal pollution in the surface soil of a region that has undergone three decades of intense industrialization and urbanization, Environmental Science pollution Research Vol 20:6 150-6159

ix. HydroAnalysis, 2015. EnviroInsite 9.2.0.44, Massachusetts: HydroAnalysis, Inc.

x. Ighere, E. J., Sogbaike, E.C., Emudianughe, P. and Mayor, A. (2014) A survey of the physiochemical characteristics of River Jamieson, Delta State Nigeria (part 1). International Journal of Scientific and Engineering Research. Vol 5, issue 1,309 .

xi. Issa, B.R., Arimoro, F.O., Ibrahim, M., Birma, G.J. and Fadairo, E.A. (2010). Assessment of Sediment Contamination by Heavy Metals in River Orogoodo, Agbor, Delta State, Nigeria. International Journal of Scientific and Technology Research. Volume 3, pp. 120-130

xii. Kaiser, H. F., 1960. The application of electronic computers to factor analysis. Educ. Psychol Meas, Volume 20, pp. 141-151.

xiii. Kaizer, A.N., and Osakwe, S.A. (2010). Physiochemical Characteristics and heavy metal levels in water samples from five river systems in Delta State, Nigeria. Journal of Applied Science and Environmental Management 14(1)83-87

xiv. Knox, G. J. \& Omatsola, M. E., 1987. Development of the Cenozoic Niger Delta in terms of the escalator regression model. In: s.l.: Kluwer Academic Publishers, p. 181-202.

xv. Kogbe, C. A., 1989. Geology of Nigeria. 2nd ed.Jos: Rockview Nige Ltd.

xvi. Krantzberg, G. et al., 2010. Advances in Water Quality Control. 1st ed. California: Scientific Research Publishing, Inc.

xvii. Meyer, G., 1988. Historical perspective. Hydrogeology: Boulder, Colorado, Geological Society of America, The Geology of the North America, Volume O-2, pp. 4-5.

xviii. Nwankwoala, H. O. \& Ngah, S. A., 2014. Quality of Groundwater in Deep Aquifer Systems of the Niger Delta. Int. J. Water Res. Environ. Eng., 6(5), pp. 155-163.

xix. Obaje, N. G., 2009. Geology and Mineral Resources of Nigeria. In: 1st ed. London: Springer, pp. 109-113.

xx. Offodile, M. E., 1991. An approach to groundwater study and development in Nigeria. In: Jos, Nigeria: Mecon Services Ltd., p. 245.

xxi. Olabaniyi, S. B. \& Owoyemi, F. B., 2006. Characterization by factor analysis of the chemical facies of groundwater in the Deltaic Plain Sands Aquifer of Warri, western Niger Delta, Nigeria. African Journal of Science and Technology (AJST) Science and Engineering Series, 7(1), pp. 73-81.

xxii. Olabaniyi, S. B. and Efe S.I. (2007). Comparative assessment of rainwater and groundwater quality in an oil producing area of Nigeria: Environmental and health implications. Journal of Environmental health Research 6(2): 111-118

xxiii. Ow oyemi, A. O., 2004. Sequence stratigraphy of Niger Delta, Delta field, offshore Nigeria. Master's thesis, Texas A\&M University, pp. 1-2.

xxiv. Piper, A. M., 1944. A graphic procedure in geochemical interpretation of water analyses.

xxv. Trans. Am. Geophys. Union, Volume 25, pp. 914-923.

xxvi. Reijers, T., 2011. Stratigraphy and sedimentology of the niger delta. Geologos, Volume 17, pp. 133-142.

xxvii. Short, K. C. \& Stauble, A. J., 1967. Outline of the geology of the Niger delta. Bull. AAPG,

xxviii. Issue 51, pp. 761-779.

xxix. Stiff, H. A. J., 1951. The interpretation of chemical water analysis by means of patterns.

xxx. Journal of Petroleum Technology, 3(10), pp. 15-17.

xxxi. Todd, D. k. \& Mays, L. W., 2005. Groundwater Hydrology. In: 3rd ed. s.l.: John Wiley \& Sons, pp. 359-366, 329-340.

xxxii. Wigwe, G. A., 1975. The Niger Delta: Physical. In: In G.E.K. Ofomata (ed). Nigeria in maps: Eastern States. Benin City: Ethiope Publ. House, pp. 380-400.

xxxiii. Yidana, S. M., 2008. Differentiating Spatial Groundwater Types in The Birimian System Using. Journal of Environmental Management, Volume 9, pp. 3-8.

xxxiv. Zaporozec, A., 1972. Graphical interpretation of water-quality data. Ground Water,10(2), p. 32-43. 